\title{
PTGER3 and MMP-2 play potential roles in diabetic nephropathy via competing endogenous RNA mechanisms
}

\author{
Yue Yu', Yuan-Yuan Jia ${ }^{2}$, Meng Wang ${ }^{3}$, Lin Mu and Hong-Jun Li ${ }^{5 *}$
}

\begin{abstract}
Background: Diabetic nephropathy (DN) is a primary complication of diabetes mellitus (DM). The pathology of DN is still vague, and diagnostic accuracy is not enough. This study was performed to identify miRNAs and genes that have possibilities of being used as therapeutic targets for DN in type 2 DM.

Methods: Human miRNA data GSE51674 and gene data GSE111154 were downloaded from the Gene Expression Omnibus database. Differentially expressed genes (DEGs) and miRNAs (DEmiRNAs) in the kidney between control and DN patients were screened out. The competing endogenous RNA (ceRNA) network was constructed, and key IncRNA-miRNA-mRNA pairs were selected accordingly. Potential drugs targeting DEGs were screened out and validated using PCR analysis.

Results: Totally, 83 DEmiRNAs and 293 DEGs were identified in GSE51674 and GSE111154, respectively. Thirteen of the top 20 DEmiRNAs (10 up and 10 down) targeted to 47 DEGs. In the ceRNA network, RP11-363E7.4/TTN-AS1/ HOTAIRM1-hsa-miR-106b-5p-PTGER3 and LINC00960-hsa-miR-1237-3p-MMP-2 interaction pairs were identified as the key ceRNA network. Interestingly, PTGER3 and hsa-miR-1237-3p were downregulated, and MMP-2 and hsa-miR-1066$5 p$ were upregulated in the kidney of patients with DN compared with normal controls, respectively. PTGER3 and MMP-2 were targeted by drugs including iloprost, treprostinil, or captopril, and the deregulation of the two genes was confirmed in the plasma samples from patients with DN as compared with controls.
\end{abstract}

Conclusions: We speculated that the RP11-363E7.4/TTN-AS1/HOTAIRM1-hsa-miR-1066-5p-PTGER3 and LINC00960-hsamiR-1237-3p-MMP-2 networks were associated with diabetic renal injury.

Keywords: Diabetic nephropathy, Microarray, microRNAs, Type 2 diabetes mellitus, Molecular biology

\section{Highlights}

1. Renal PTGER3 and miR-1237-3p were reduced in diabetic nephropathy (DN).

2. $M M P-2$ and $m i R-106 b-5 p$ were increased in the kidney of patients with $\mathrm{DN}$.

\footnotetext{
* Correspondence: hj_li@jlu.edu.cn

${ }^{5}$ Health Management Medical Center, China-Japan Union Hospital of Jilin University, 126 Xiantai Street, Changchun 130033, Jilin Province, China

Full list of author information is available at the end of the article
}

3. PTGER3 reduction and MMP-2 upregulation may protect renal injury from diabetes.

\section{Background}

Diabetes mellitus (DM) is a metabolic disorder that affects 1 in 11 adults aged between 20 and 79 years old in 2015 [1-3]. The global estimated prevalence of DM is expected to rise to 578 million by 2030 and $\sim 700$ million by $2045[4,5]$. People with DM are at high risk of developing serious problems and life-threatening health complications, including diabetic nephropathy (DN),

(c) The Author(s). 2021 Open Access This article is licensed under a Creative Commons Attribution 4.0 International License, which permits use, sharing, adaptation, distribution and reproduction in any medium or format, as long as you give appropriate credit to the original author(s) and the source, provide a link to the Creative Commons licence, and indicate if changes were made. The images or other third party material in this article are included in the article's Creative Commons licence, unless indicated otherwise in a credit line to the material. If material is not included in the article's Creative Commons licence and your intended use is not permitted by statutory regulation or exceeds the permitted use, you will need to obtain permission directly from the copyright holder. To view a copy of this licence, visit http://creativecommons.org/licenses/by/4.0/ The Creative Commons Public Domain Dedication waiver (http://creativecommons.org/publicdomain/zero/1.0/) applies to the data made available in this article, unless otherwise stated in a credit line to the data. 
retinopathy, cardiovascular disease, and stroke [1, 6-8]. $\mathrm{DN}$ is the primary cause of end-stage renal failure and chronic kidney disease [6]. It is a major chronic complication that affects over $40 \%$ of DM and $\sim 10 \%$ of type 2 DM died of renal failure globally $[1,3,9,10]$.

The pathophysiology leading to $\mathrm{DN}$ and resultant renal failure from DM consists of hypertension, altered composition and proliferation, and sclerosis in glomerular [10]. Accordingly, the estimated glomerular filtration rate (eGFR), albuminuria, and hypertension are the clinical manifestations of $\mathrm{DN}$ [3]. However, the clinical usage of them could not make an accurate and definite diagnosis of DN and the degree of kidney damage [3]. Herein, DN is often confirmed during postmortem examination because of the lack of definitive clinical findings. Theoretically, the progression and complications of $\mathrm{DN}$ could be blocked or slowed down by earlystage interventions [10-12]. A major obstacle for that is the lack of accurate biomarkers identifying patients who at high risk of DN. To fulfill this gap, experiments are being carried out to find effective methods for the accurate and preoperative diagnosis of DN [13].

Much evidence has shown that genetic factors are the major predispositions to type $2 \mathrm{DM}$ and $\mathrm{DN}[1,10]$. Many non-coding RNAs and genes like transforming growth factor (TGF) $\beta$, nephrin, angiotensin-converting enzyme-2, interleukin-6 (IL-6), and tumor necrosis factor (TNF)- $\alpha$ have been identified to be associated with the development, progression, and prognosis of DN [9, 12-14]. These genetic factors modulate extracellular matrix structure as well as the oxidative, fibrotic, and inflammatory responses in the kidney, tubular, and glomerular $[12,15,16]$. Nevertheless, no single treatment targeting the above factors has been able to reverse or mitigate $\mathrm{DN}$ progression, or reduce $\mathrm{DN}$-associated mortality [14]. Therefore, additional factors that have therapeutic targeting possibilities in $\mathrm{DN}$ are in urgent need.

This study was performed to identify the genes and miRNAs that were differentially expressed in the kidney of patients with $\mathrm{DN}$. Integrated bioinformatics analysis was performed for screening the miRNAs and genes in DN in type 2 DM. Moreover, the possibility of using them as therapeutic targets would be discussed.

\section{Methods}

\section{Microarray data}

The human miRNA microarray dataset GSE51674 and gene expression dataset GSE111154 were downloaded from the National Center for Biotechnology Information (NCBI) Gene Expression Omnibus (GEO, http://www. ncbi.nlm.nih.gov/geo/). GSE51674 (GPL10656 Agilent029297 Human miRNA Microarray v14 Rev.2; miRNA ID version) consisted of 10 kidney samples from 4 controls (normal kidney from patients underwent kidney biopsy) and $6 \mathrm{DN}$ patients (kidney tissues from patients with type $2 \mathrm{DM}$ for $15 \pm 7$ years and biopsy-proven $\mathrm{DN}$ ) [6]. GSE111154 (GPL17586 [HTA-2_0] Affymetrix Human Transcriptome Array 2.0, transcript (gene) version) consisted of 8 kidney cortical tissue samples from 4 non-diabetic controls and $4 \mathrm{DN}$ patients (type $2 \mathrm{DM}$ with early DN) [3].

\section{Data processing}

The raw data in GSE51674 and GSE111154 datasets were downloaded and processed using the Limma package [17] and Oligo package [18], respectively. Data processing included background correction, normalization, and expression calculation. The probes in GSE51674 and GSE111154 that mapped to human miRNAs and mRNAs, respectively, were retained and used for further analysis. When multiple probes mapped to one gene symbol, the averaged expression level of probes was calculated and regarded as the gene expression level of that gene or miRNA. The probes that did not map to genes or human miRNAs were removed.

\section{Differential expression analysis}

The differentially expressed genes (DEGs) and miRNAs (DEmiRNAs) between control and disease samples were identified using the paired $\mathrm{t}$-test in the Limma package [17]. Significant DEGs were identified according to the criteria of $p$ value $<0.05$ and $\mid \log _{2}$ (fold change, $\left.\mathrm{FC}\right) \mid>$ 0.585 , and significant DEmiRNAs were screened out according to stricter criteria of $\left|\log _{2} \mathrm{FC}\right|>2$ and adjusted (BH correction) $\mathrm{p}$ value $<0.05$.

\section{Construction of protein-protein interaction (PPI) network}

The protein-protein interaction (PPI) pairs among the products of DEGs were screened in the STRING (Version 10.0, http://www.string-db.org/) [19]. The interaction pairs with a score of higher than 0.4 (medium confidence) were retained and used for the construction of the PPI network. Cytoscape (version 3.2.0, http://www.cytoscape.org/) was employed for the construction of the PPI network. Dominant modules in the PPI network were identified using the MCODE plugin (version 1.4.2, http://apps.cytoscape. org/apps/MCODE) that was provided by Cytoscape. The DEGs in modules were employed for the enrichment analysis.

\section{Functional enrichment analysis}

The functional enrichment analysis was performed separately for the DEGs. The clusterprofiler in $\mathrm{R}$ was used for enriching the Gene Ontology biological processes and Kyoto Encyclopedia of Genes and Genomes (KEGG) pathways. Significant enrichment was identified with the thresholds of adjusted (BH correction) $p$ value $<0.05$ and gene number count $\geq 2$. 


\section{Prediction of miRNA-mRNA target}

To investigate the correlation between the DEmiRNAs and DEGs in DN, the predictive targets of DEmiRNAs were screened from the DEG list and the miRNAmRNA regulatory network was constructed accordingly. The targets that were collected by at least 6 of the 7 databases (miRWalk, miRanda, miRDB, miRMap, Pictar2, RNA22, and Targetscan) were regarded as potential targets in our study. Then, the miRNA-mRNA target pairs were obtained and used for the construction of the miRNA-mRNA regulatory network using Cytoscape.

\section{Prediction of IncRNAs that sponge DEmiRNAs}

To investigate the competing endogenous RNA (ceRNA) involving lncRNAs, DEGs, and DEmiRNAs in DN, the potential IncRNA-miRNA pairs were identified in the DIANA-LncBase V2 [20]. The lncRNAs that tightly interacted with the DEmiRNAs (score $\geq 0.99$ ) in the kidney were selected. The miRNA-mRNA and lncRNA-miRNA interaction pairs that are linked by the same miRNAs were retained and used for the construction of the IncRNAmiRNA-mRNA (ceRNA) network. Cytoscape was used for the construction of the ceRNA network.

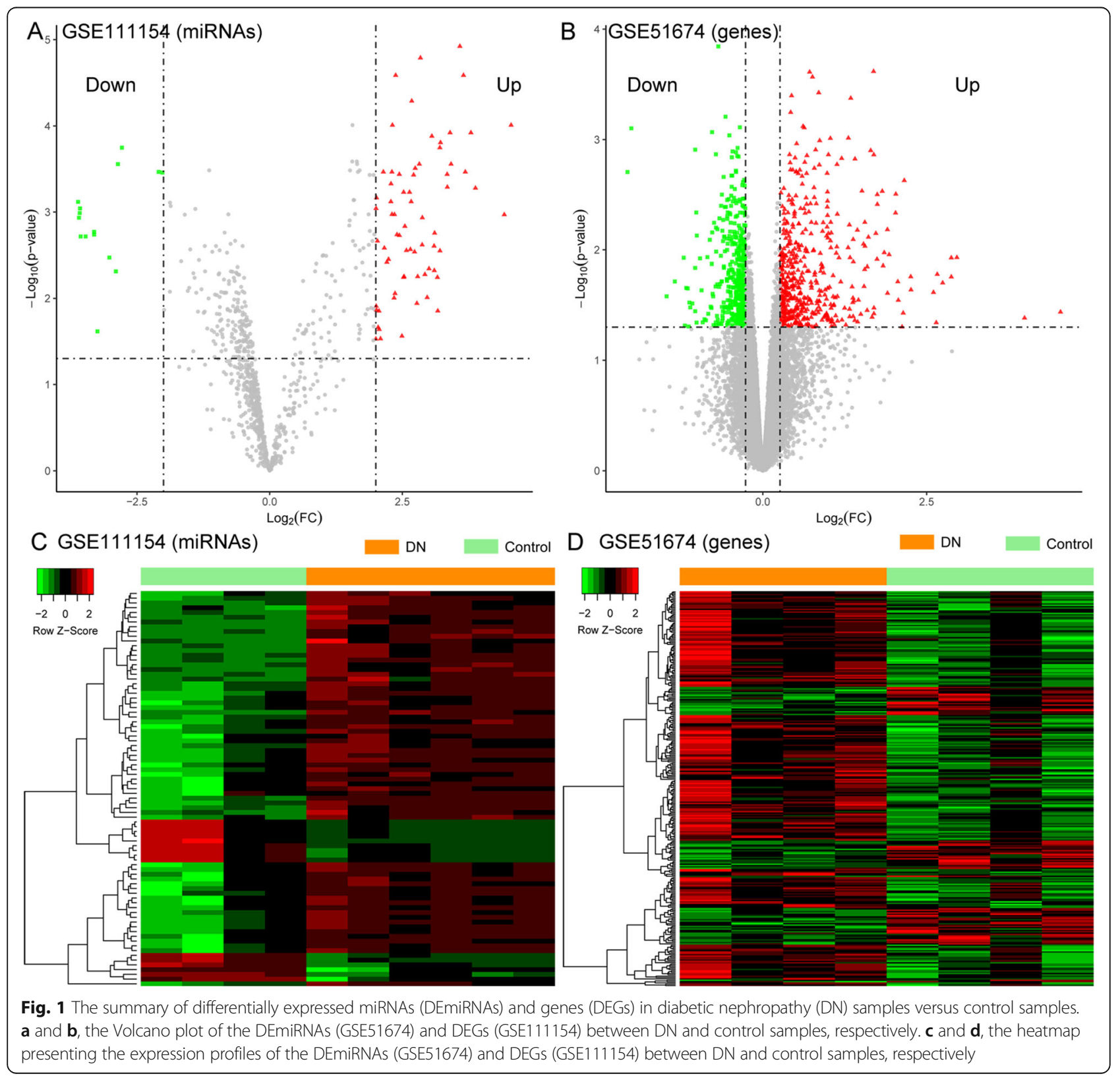




\section{Drug-gene interaction network}

Drug-Gene Interaction database (DGIdb) provides information on the interactions between drugs and genes, which might be of great value for settling potential therapeutic strategy that targets the key genes. DGIdb was employed to extract the drugs targeting the DEGs in the above ceRNA network. Drugs that have been admitted by the Food and Drug Administration (FDA) were reserved and used to construct the drug-gene interaction network.

\section{Patients and ethical statement}

The peripheral blood samples were collected from three patients with $\mathrm{DN}$ and three healthy controls from the

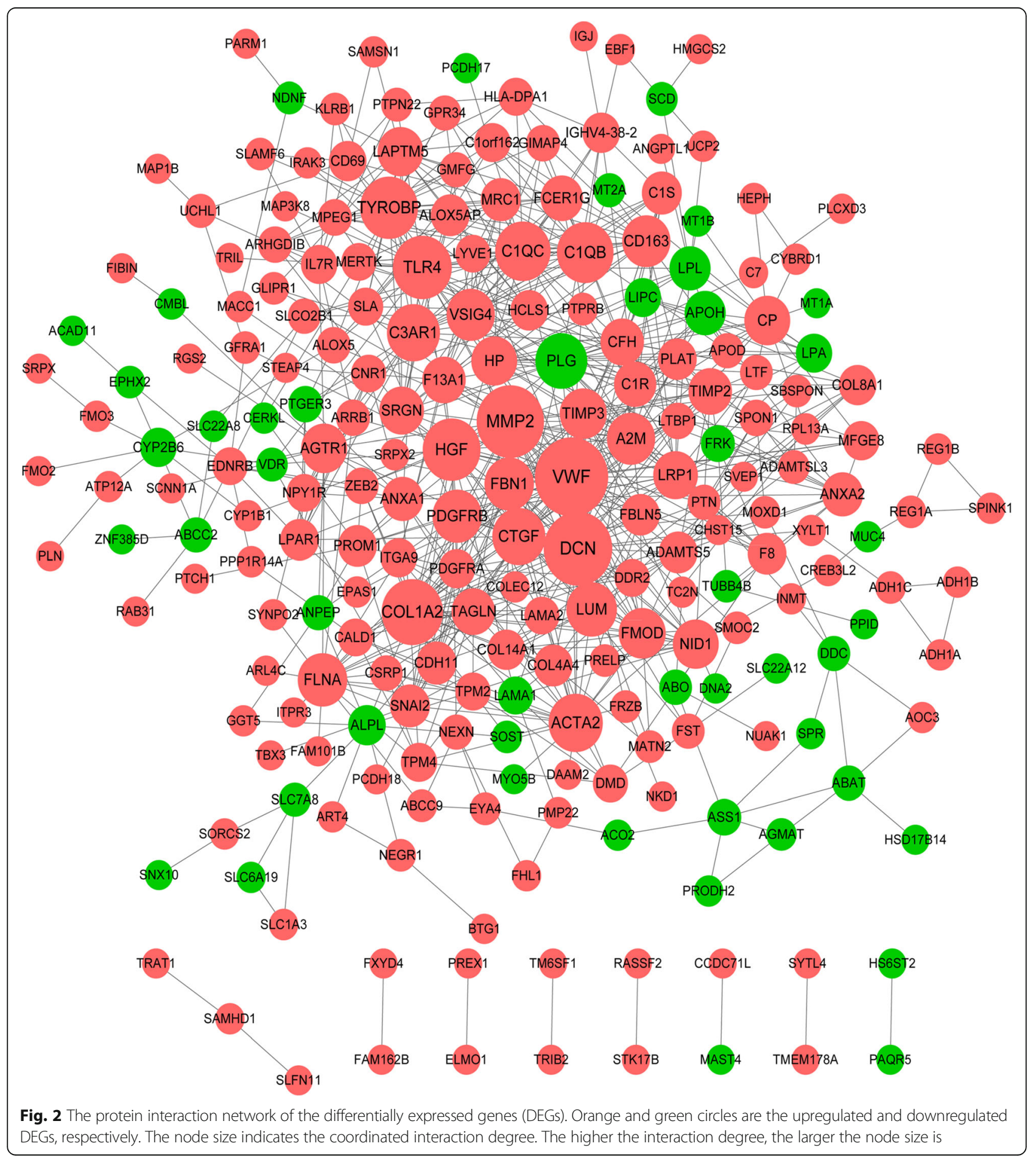


Department of Endocrinology, China-Japan Union Hospital of Jilin University, Changchun, China, in Sept 2020. Approval was obtained from the Ethics Committee of China-Japan Union Hospital of Jilin University, and written informed consent was obtained from all participants before the collection of blood samples. All the samples were stored at $4{ }^{\circ} \mathrm{C}$ before RNA extraction.

\section{RNA isolation and PCR amplification}

The total RNA was isolated from the plasma samples using RNAiso Plus (Trizol; TaKaRa, Tokyo, Japan; \#9109). Reverse transcription into cDNA was performed using a PrimeScript ${ }^{\mathrm{m}}$ II 1 st Strand cDNA Synthesis Kit (TaKaRa, \#6210A). The real-time quantitative PCR amplification was performed using the Power SYBR Green PCR Master Mix (Thermo Fisher Scientific, Applied Biosystems, \#4367659). The reaction conditions were: $50^{\circ} \mathrm{C}$ for $2 \mathrm{~min}$; $95^{\circ} \mathrm{C}$ for $2 \mathrm{~min}$; 40 cycles of $95^{\circ} \mathrm{C}$ for $15 \mathrm{~s}$, $60^{\circ} \mathrm{C}$ for $60 \mathrm{~s}$. The primers used for the amplification of PTGER3 were: forward 5' - CGCCTCAACCACTCCTAC AC-3', reverse 5' - GACACCGATCCGCAATCCTC-3', and the primers used for the amplification of $M M P-2$ were: forward 5' - TACAGGATCATTGGCTACACAC C-3', and reverse 5' - GGTCACATCGCTCCAGACT-3'.
The primers used for the amplification of the internal reference gene GAPDH were: forward 5' - TGACAACT TTGGTATCGTGGAAGG-3', and reverse 5'- AGGCAGGGATGATGTTCTGGAGAG-3'. The relative expression levels of MMP-2 and PTGER3 were calculated using the $2^{-\triangle \Delta \mathrm{Ct}}$ methods.

\section{Statistical analysis}

The expression levels of the MMP-2 and PTGER3 genes were expressed as mean \pm standard deviation. The differences in the expression levels of MMP-2 and PTGER3 were analyzed using the t-test in Graphpad Prism 5 (Graphpad Software, San Diego, CA). $P<0.05$ was set as the threshold for the significant difference.

\section{Results}

Identification of DEmiRNAs and DEGs

After data normalization, a total of 83 DEmiRNAs $\left(\left|\log _{2} \mathrm{FC}\right|>2\right.$ and adjusted $\left.p<0.05\right)$ and 293 DEGs $(\mathrm{p}<$ 0.05 and $\left.\left|\log _{2} \mathrm{FC}\right|>0.585\right)$ were identified in the GSE51674 dataset and the GSE111154 dataset, respectively (Fig. 1a and b). The sample clusterings based on these DEmiRNAs and DEGs are presented in Fig. 1c and $\mathrm{d}$, respectively. The heatmaps indicated that expression

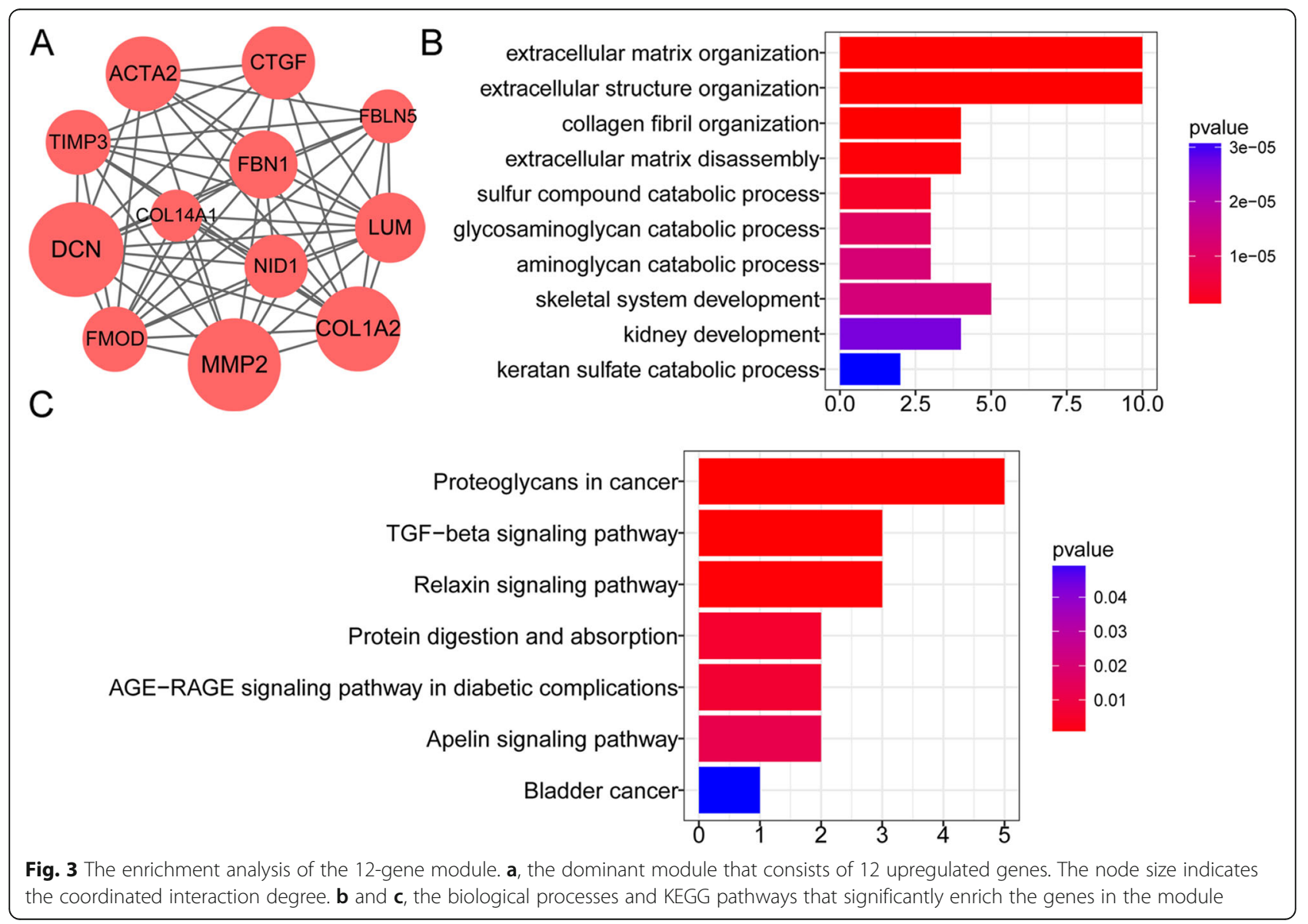


profiles of these genes were different between the DN and control samples.

\section{Analysis of the PPI network}

Based on the predicted protein interactions in the STRI NG database, the PPI network that consisted of 228 DEGs and 732 interaction pairs was constructed (Fig. 2). A dominant module with a score of 10.364 was identified (Fig. 3a). This module was composed of 12 products of upregulated genes and 57 interaction pairs. In this module, MMP-2, COL1A2, ACTA2, and CTGF had high interaction degrees of $34,29,23$, and 22 , respectively. These DEGs in the module were involved in biological processes like 'extracellular matrix organization', 'extracellular matrix disassembly', 'sulfur compound catabolic process', 'glycosaminoglycan catabolic process', and 'kidney development' (Fig. 3b), and KEGG pathways like 'TGF-beta signaling pathway', 'Relaxin signaling pathway', 'AGE-RAGE signaling pathway in diabetic complications', and 'Apelin signaling pathway' (Fig. 3c).

\section{Construction of the miRNA-mRNA regulatory network}

The miRNA-mRNA regulatory network was constructed to investigate the regulatory mechanisms involving the DEmiRNAs and DEGs in DN. Before the prediction of miRNA targets, 10 upregulated and 10 downregulated DEGs with the top absolute values of $\log _{2} \mathrm{FC}$ were selected (Table 1). Then, a total of 47 DEGs (42 up- and 5 downregulated DEGs) were identified to be targeted by 13 DEmiRNAs, including 10 upregulated DEmiRNAs and 3 downregulated DEmiRNAs. Subsequently, the differentially expressed miRNA-mRNA network was constructed (Fig. 4). In this network, 17, 7, and 6 DEGs were targeted by hsa-miR-106b-5p, hsa-miR-374a-5p, and $h s a-m i R-1237-3 p$, respectively.

\section{Construction of the IncRNA-miRNA-mRNA regulatory network}

The ceRNA network was constructed using the 6 miRNAs had interaction degrees of greater than 5 in the miRNAmRNA network, including hsa-miR-106b-5p, hsa-miR374a-5p, hsa-miR-1237-3p, hsa-miR-19a-3p, hsa-miR$34 a-5 p$, and $h s a-m i R-374 b-5 p$. Eighteen lncRNA-miRNA pairs had scores of higher than 0.99 were screened out accordingly. After integrating the aforementioned miRNAmRNA and lncRNA-miRNA pairs, 63 lncRNA-miRNAmRNA pairs were screened out. The corresponding ceRNA network was composed of 63 lncRNA-miRNAmRNA pairs, 6 DEmiRNAs, 33 DEGs, and 17 lncRNAs (Fig. 5). In this network, hsa-miR-106b-5p, hsa-miR-1237$3 p$, and $h s a-m i R-374 a-5 p$ had a relative higher interaction degree of 23, 11 and 10, respectively. The potential ceRNA networks included RP11-363E7.4/TTN-AS1/HOTAIRM1-
hsa-miR-106b-5p-PTGER3 and LINC00960-hsa-miR1237-3p-MMP-2.

\section{Identification of potential drug targets}

To understand the potential drugs targeting PTGER3 and $M M P-2$, the drug-gene interactions were predicted and the gene-drug interaction network involving the two genes was constructed (Fig. 6). It was composed of 6 PTGER3 agonists (bimatoprost, dinoprostone, misoprostol, iloprost, dinoprost, and treprostinil) and two $M M P-2$ inhibitors (captopril and tiludronate; Fig. 6).

\section{Validation of the MMP-2 and PTGER3 expression}

The PCR analysis showed that there were significant differences in the expression levels of the $M M P-2$ and PTGER3 genes in the plasma samples between patients with DN and healthy controls (Fig. 7a and b). The expression level of the PTGER3 gene in patients with DN was significantly lower than that from healthy controls ( $p=0.0360$, Fig. 7a), while the $M M P-2$ gene was significantly upregulated in DN patients as compared with controls $(p=0.0054$, Fig. $7 \mathrm{~b})$.

Table 1 The list of the top 20 differentially differentially expressed miRNAs in diabetes versus controls

\begin{tabular}{llll}
\hline miRNA & logFC & P.Value & adj. P.Value \\
\hline Top 10 downregulated miRNAs & & \\
hsa-miR-223-3p & 4.55 & $8.49 \times 10^{-7}$ & $9.77 \times 10^{-5}$ \\
hsa-miR-150-5p & 4.42 & $7.13 \times 10^{-5}$ & $1.07 \times 10^{-3}$ \\
hsa-miR-142-3p & 3.88 & $2.26 \times 10^{-5}$ & $5.27 \times 10^{-4}$ \\
hsa-miR-34a-5p & 3.79 & $1.22 \times 10^{-6}$ & $1.20 \times 10^{-4}$ \\
hsa-miR-19a-3p & 3.69 & $1.12 \times 10^{-5}$ & $3.42 \times 10^{-4}$ \\
hsa-miR-374b-5p & 3.65 & $9.34 \times 10^{-8}$ & $2.60 \times 10^{-5}$ \\
hsa-miR-146b-5p & 3.58 & $1.35 \times 10^{-8}$ & $1.19 \times 10^{-5}$ \\
hsa-miR-106b-5p & 3.42 & $6.17 \times 10^{-6}$ & $2.77 \times 10^{-4}$ \\
hsa-miR-374a-5p & 3.40 & $1.35 \times 10^{-6}$ & $1.20 \times 10^{-4}$ \\
hsa-miR-497-5p & 3.35 & $2.14 \times 10^{-5}$ & $5.13 \times 10^{-4}$ \\
Top 10 upregulated miRNAs & & $1.93 \times 10^{-3}$ \\
hsa-miR-1237-3p & -3.02 & $3.88 \times 10^{-4}$ & $3.38 \times 10^{-3}$ \\
hsa-miR-1973 & -3.25 & $6.97 \times 10^{-3}$ & $2.42 \times 10^{-2}$ \\
hsa-miR-1281 & -3.31 & $1.26 \times 10^{-4}$ & $1.70 \times 10^{-3}$ \\
hsa-miR-1225-3p & -3.31 & $1.46 \times 10^{-4}$ & $1.83 \times 10^{-3}$ \\
hsa-miR-425-3p & -3.47 & $1.60 \times 10^{-4}$ & $1.92 \times 10^{-3}$ \\
hsa-miR-1825 & -3.56 & $1.60 \times 10^{-4}$ & $1.92 \times 10^{-3}$ \\
hsa-miR-1234 & -3.57 & $5.06 \times 10^{-5}$ & $9.08 \times 10^{-4}$ \\
hsa-miR-1238 & -3.58 & $6.02 \times 10^{-5}$ & $1.03 \times 10^{-3}$ \\
hsa-miR-191-3p & -3.59 & $8.00 \times 10^{-5}$ & $1.16 \times 10^{-3}$ \\
\hline
\end{tabular}




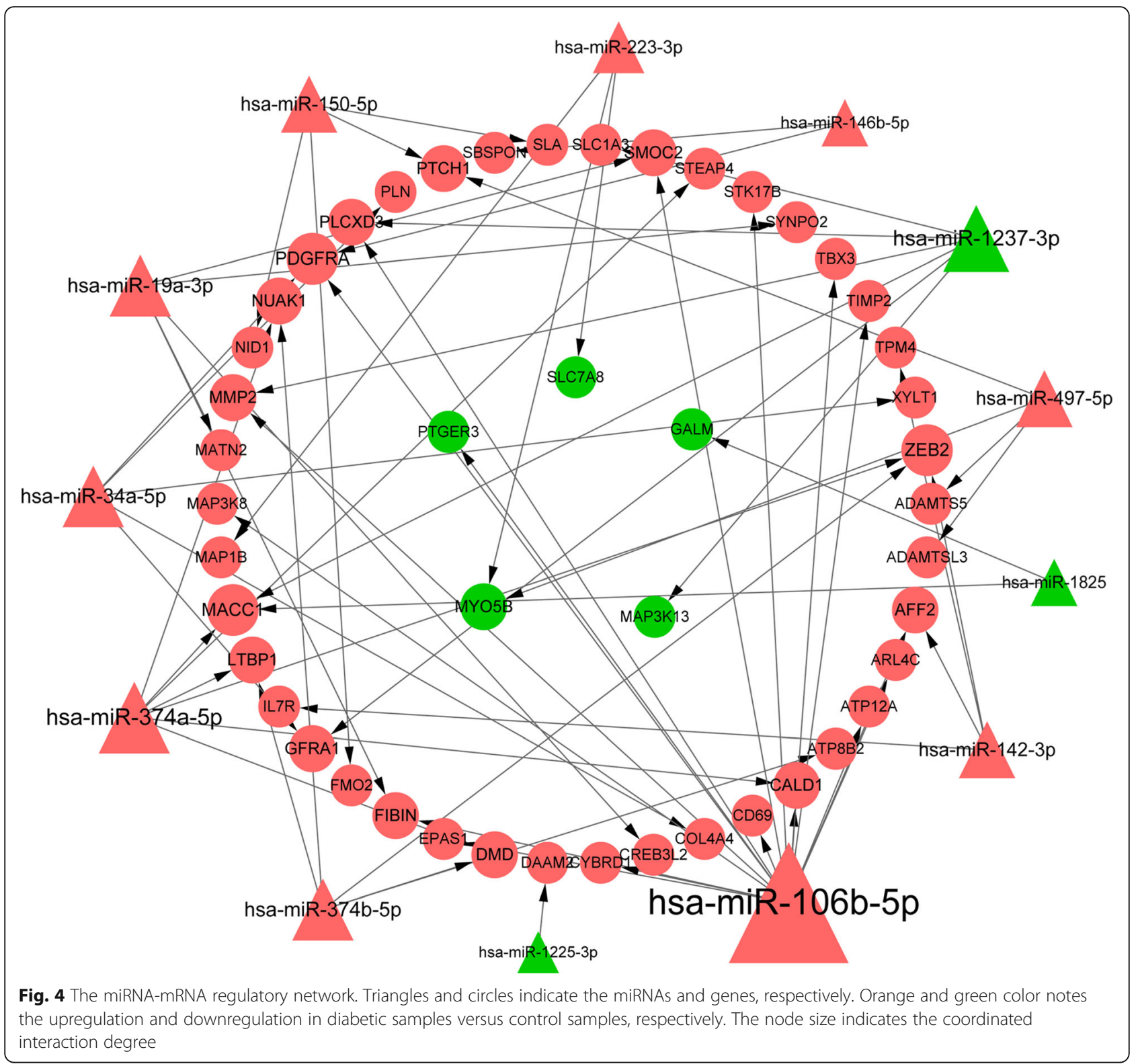

\section{Discussion}

Based on the integrated bioinformatics analysis using GSE51674 and GSE111154 datasets, we finally identified two potential therapeutic targets in DN, including downregulated PTGER3 and hsa-miR-1237-3p, upregulated $M M P-2$ and $h s a-m i R-106 b-5 p$. In addition, ceRNA interactions including RP11-363E7.4/TTN-AS1/HOTAIRM1hsa-miR-106b-5p-PTGER3 and LINC00960-hsa-miR1237-3p-MMP-2 might be of novel and great value for clearing the pathogenesis and progression of $\mathrm{DN}$. We also identified the therapeutic possibilities of targeting PTGER3 and MMP-2.

The member of the EP receptor family, prostaglandin EP3 receptor gene (PTGER3/EP3) is a G protein-coupled receptor that facilitates a broad range of physiological actions including blunting glucose-stimulated insulin secretion (GSIS) [21]. It has been linked to the dysfunction and loss of $\beta$-cells in type 2 DM [21, 22]. PTGER3 upregulation in the islets of patients with type $2 \mathrm{DM}$ has been proven previously [23]. Additionally, elevated production of prostaglandin $E_{2}$ (PE2) and PTGER3 can be targeted to improve insulin secretion from islets [24]. Pathologically, PTGER3 negatively regulates the production of cyclic AMP (cAMP), a potentiator of GSIS [22, 25]. Rankin et al. [21] showed that agonist (sulprostone) and antagonist of EP3r both significantly reduced GSIS and fasting plasma glucagon in non-human primates with noninsulin-dependent diabetes mellitus. Schaid et al. [24] showed that $\gamma$ PTGER3 was constitutively activated in pancreatic $\beta$-cells and its action of reducing 


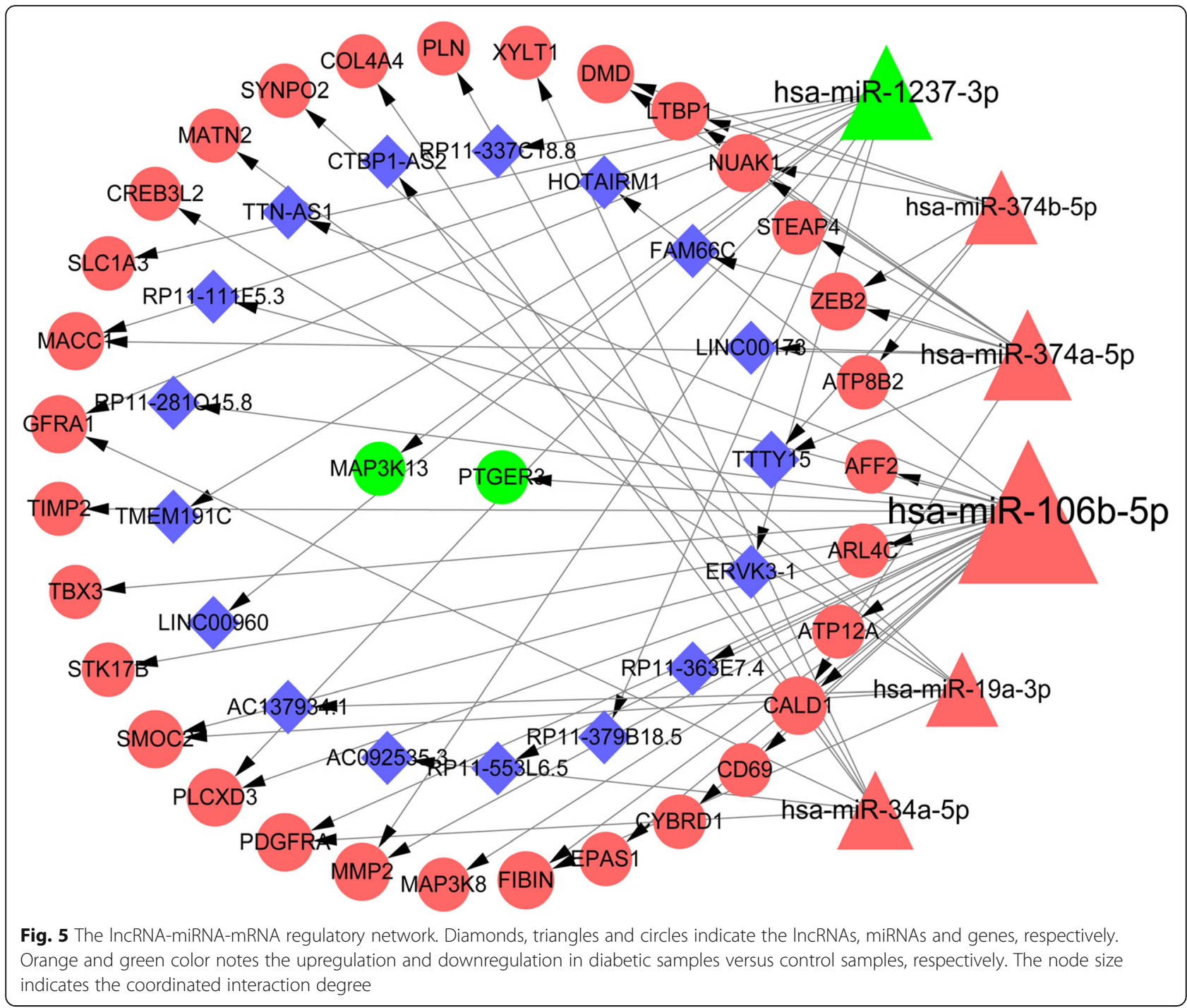

insulin was Goz dependent. The $\alpha$ PTGER3 activity, however, could be stimulated by sulprostone in Gaz/ cAMP-dependent and -independent signalings. After treatment with sulprostone, Rap1GAP-mediated inhibition of GSIS was potentiated in $\beta$-cells [24]. This was in line with the results reported by Rankin et al. [21].

However, the association of PTGER3 expression with nephropathy or other renal diseases is rarely reported [3, $26,27]$. In renal cell carcinoma (RCC), the lower expression of PTGER3 was correlated with a worse prognosis [26]. In patients with DN, downregulation of PTGER3 in the kidney from donors with early DN versus matched controls has been confirmed by Sircar et al. [3]. What's more, there was a differentially methylated level in the PTGER3 gene between diabetic patients with end-stage renal disease and those without nephropathy [27]. Two or more methylated CpG sites were found in the PTGE $R 3$ gene in diabetic patients with the end-stage renal disease compared with those without nephropathy [27]. An in vivo animal experiment showed that the knockout of PTGER3 did not reduce the levels of plasma and urine glucose in the streptozotocin-induced mouse model of diabetes [28]. However, there was a significant increment in urine osmolality, water reabsorption, and aquaporins expression in mice kidney. Obvious reductions in renal hypertrophy, hyperfiltration, albuminuria, tubular dilation, and nuclear cysts were observed in the kidney in streptozotocin-treated $E p_{3}{ }^{-1-}$ mice compared with wild type mice [28]. These results showed that PTGER3 downregulation was a specific protection for the kidney against metabolic disorders in diabetes. Its downregulation might be used as a biomarker or therapeutic strategy for DN.

Apart from the PTGER3 gene, matrix metalloproteinase-2 $(M M P-2)$ is also differentially methylated in diabetic patients with the end-stage renal disease compared with those 

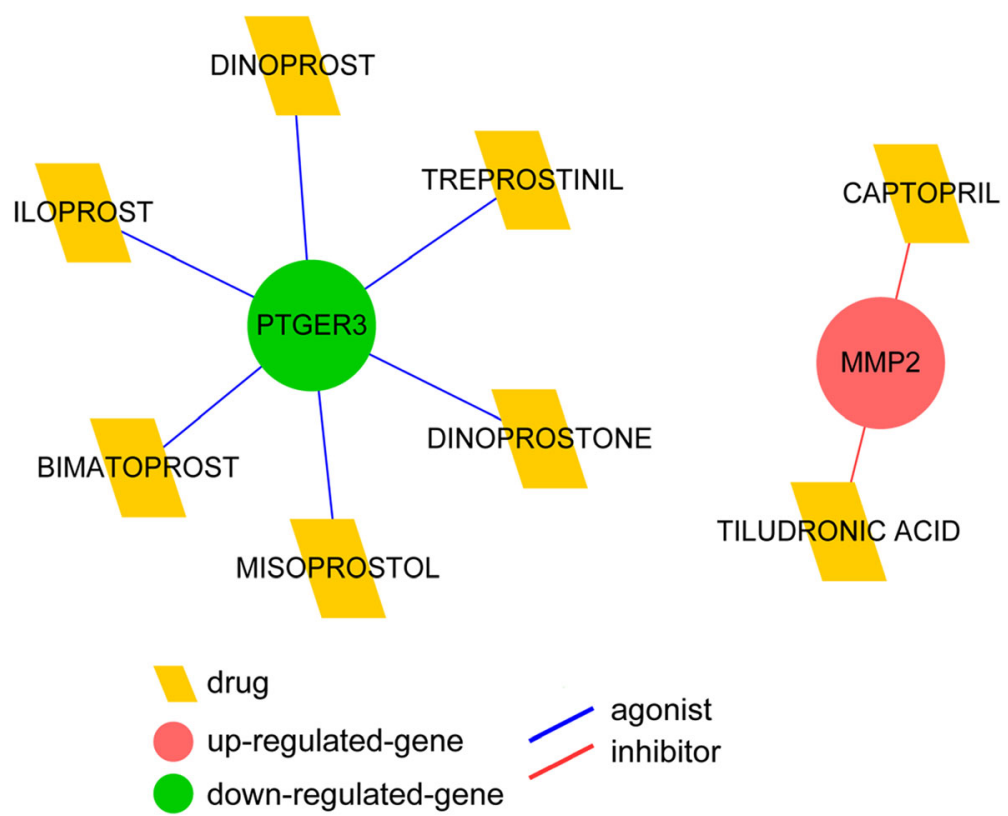

Fig. 6 The drug and gene interaction network involving two key candidates

without nephropathy [27]. MMP-2 is a well-characterized protein responsible for tissue remodeling and extracellular matrix degradation. MMP-2 expression is a marker of newly formed $\beta$-cells and is negative in the islets of adult rats [29]. It was positively correlated with periodontitis severity in patients with type $2 \mathrm{DM}$ [30]. In addition, the expression of $M M P$-2 was higher in patients with type $2 \mathrm{DM}$ compliant with the peripheral arterial disease compared with diabetic patients without arterial diseases and control groups [31,32]. In patients with chronic kidney diseases, creatinine level was positively correlated with $M M P-2(\mathrm{r}=0.39)$, but in diabetic patients it was negative correlation $(r=-0.72)$ [31]. Our present study confirmed the upregulation of $M M P-2$ in $\mathrm{DN}$ might be associated with the remodeling of renal and preventing sclerosis in glomerular. The potential of employing if as a biomarker for $\mathrm{DN}$ in patients with diabetes should be validated.

Based on the analysis of DEmiRNAs in DN, we identified the potential regulatory networks in $\mathrm{DN}$, including RP11-363E7.4/TTN-AS1/HOTAIRM1-hsa-miR-106b-5pPTGER3 and LINC00960-hsa-miR-1237-3p-MMP-2. In direct contrary to PTGER3 and MMP-2, hsa-miR-106b$5 p$ and $h s a-m i R-1237-3 p$ were up- and down-regulated in $\mathrm{DN}$ versus control, respectively. Hsa-miR-106b-5p has been proven to contribute to $\beta$-cell proliferation following bone marrow transplantation [33]. It was also
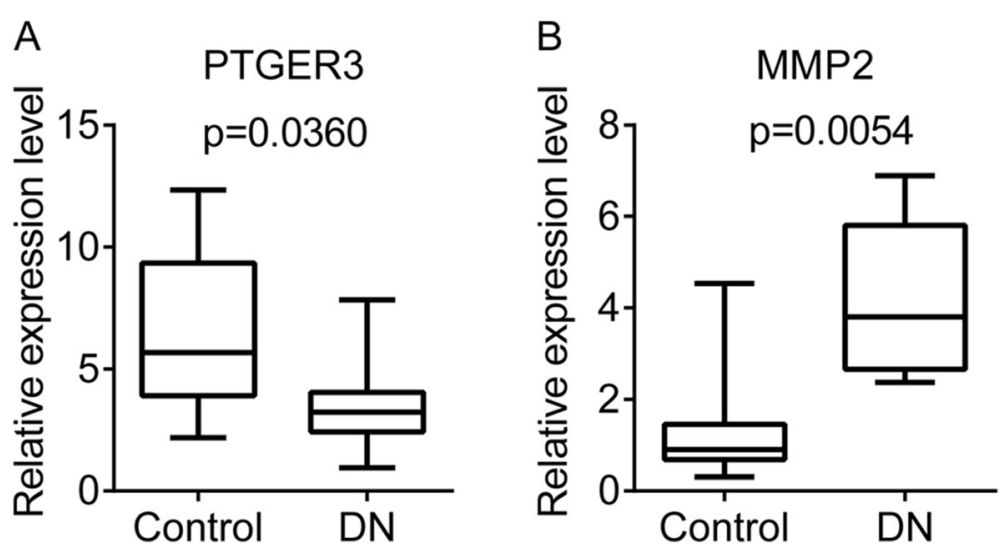

Fig. 7 The expression of the two genes in the plasma samples from patients with diabetic nephropathy (DN). $\mathbf{a}$ and $\mathbf{b}$, the expression levels of the PTGER3 gene and the MMP-2 gene in the plasma samples from patients with DN, respectively. The difference was analyzed using the t-test 
decreased in diabetic patients with chronic kidney disease versus patients without kidney disease [34], and is significantly linked to kidney function and eGFR [35]. HOTAIRM1 expression is crucial for the differentiation of embryonic stem cells to renal lineage [36]. However, there is less information on the association of these miRNAs and lncRNAs with DN and the progression of other renal diseases. The tight interactions between these lncRNAs, DEmiRNAs (hsa-miR-106b-5p and $h s a-m i R-$ 1237-3p), and DEGs (including PTGER3 and MMP-2) indicated the potential and important roles of them in regulating kidney function.

Here in our study, we identified that PTGER3 and $M M P$ - 2 were targeted by drugs including iloprost, treprostinil, and captopril. Iloprost and treprostinil are clinically used for severe pulmonary arterial hypertension and chronic thromboembolic pulmonary hypertension $[37,38]$. Iloprost shows efficacy in decreasing urinary albumin excretion rate in patients with $\mathrm{DN}$ and attenuating the progression of $\mathrm{DN}$ [39]. Iloprost could downregulate $M M P-2$ via direct targeting [40]. Since the downregulation of PTGER3 and upregulation of MMP-2 are protective for renal diseases, the agonists of PTGER3 (including iloprost and treprostinil) and inhibitor of MMP-2 (like captopril) might be inapplicable for the treatment of DN. Novel drugs aiming to increase $M M P$ 2 or decrease PTGER3 in the kidney might of great values for preventing $\mathrm{DN}$ and renal damages in diabetes.

\section{Conclusions}

In summary, we confirmed the crucial roles of PTGER3 downregulation and $M M P-2$ upregulation in the kidney from patients with DN. They were regulated by lncRNAs (including RP11-363E7.4, TTN-AS1, HOTAIRM1, and LINC00960) and miRNAs (including hsa-miR-106b-5p and $h s a-m i R-1237-3 p$ ) that had been rarely reported in DN. Their dysregulation might be the defense mechanism against renal injury following diabetes. However, more preclinical experiments and clinical trials should be performed to determine the dysregulation of them was a blessing or a curse for $\mathrm{DN}$.

\footnotetext{
Abbreviations

DM: Diabetes mellitus; DN: Diabetic nephropathy; eGFR: Estimated glomerular filtration rate; GEO: Gene Expression Omnibus; DEGs: Differentially expressed genes; DEmiRNAs: Differentially expressed miRNAs; KEGG: Kyoto Encyclopedia of Genes and Genomes; PPI: Protein-protein interaction; ceRNA: Competing endogenous RNA; DGldb: Drug-Gene Interaction database; FDA: Food and Drug Administration; GSIS: Glucose-stimulated insulin secretion; PE2: Prostaglandin E2; CAMP: Cyclic AMP; PTGE R3: Prostaglandin EP3 receptor gene; RCC: Renal cell carcinoma; MMP2: Matrix metalloproteinase-2
}

\section{Authors' contributions}

Conception and design of the research: YY, YJ, MW, LM and HL. Acquisition, analysis and interpretation of data: YY, YJ, MW and LM. Drafting the manuscript: YY. Manuscript revision for important intellectual content: HL. All authors have read and approved the manuscript.

\section{Funding \\ None.}

\section{Availability of data and materials}

The human miRNA microarray dataset GSE51674 and gene expression dataset GSE111154 are available at the National Center for Biotechnology Information (NCBI) Gene Expression Omnibus (GEO, http://www.ncbi.nlm.nih. gov/geo/). The original raw data for the PCR analysis in DN patients were available from the corresponding author with reasonable requirement.

\section{Ethics approval and consent to participate}

Approval was obtained from the Ethics Committee of China-Japan Union Hospital of Jilin University, Jilin, China. Written informed consents were obtained from all participants before the collection of blood samples.

\section{Consent for publication}

Consent for publication was obtained from each participant before and after sample collection.

\section{Competing interests}

The authors declare that they have no competing interests.

\section{Author details}

${ }^{1}$ Department of Endocrinology, China-Japan Union Hospital of Jilin University, Changchun 130033, Jilin Province, China. ${ }^{2}$ China-Japan Union Hospital of Jilin University, Changchun 130033, Jilin Province, China. ${ }^{3}$ Center of Reproductive Medicine, Center of Prenatal Diagnosis, the First Hospital of Jilin University, Changchun 130021, Jilin Province, People's Republic of China. ${ }^{4}$ Department of Radiology, The First Hospital of Jilin University, Changchun 130021, Jilin Province, People's Republic of China. ${ }^{5}$ Health Management Medical Center, China-Japan Union Hospital of Jilin University, 126 Xiantai Street, Changchun 130033, Jilin Province, China.

Received: 16 July 2020 Accepted: 29 November 2020

Published online: 12 January 2021

\section{References}

1. Zheng Y, Ley S, Hu F. Global aetiology and epidemiology of type 2 diabetes mellitus and its complications. Nat Rev Endocrinol. 2017;14:88-98.

2. Federation ID: IDF Diabetes Atlas-7th Edition. Diabetes Atlas 2015:http:// www.diabetesatlas.org/.

3. Sircar M, Rosales IA, Selig MK, Xu D, Zsengeller ZK, Stillman IE, Libermann TA, Karumanchi SA, Thadhani RI. Complement 7 is up-regulated in human early diabetic kidney disease. Am J Pathol. 2018;188(10):2147-54.

4. Yuen L, Saeedi P, Riaz M, Karuranga S, Divakar H, Levitt N, Yang X, Simmons D. IDF diabetes atlas: projections of the prevalence of hyperglycaemia in pregnancy in 2019 and beyond: results from the international diabetes Federation diabetes atlas. Diabetes Res Clin Pract. 2019;157:107841.

5. Cho NH, Shaw JE, Karuranga S, Huang Y, Rocha Fernandes JDD, Ohlrogge AW, Malanda B. IDF Diabetes Atlas: Global estimates of diabetes prevalence for 2017 and projections for 2045. Diabetes Res Clin Pract. 2018;138:271-81. https://doi.org/10.1016/j.diabres.2018.1002.1023.

6. Conserva F, Barozzino M, Pesce F, Divella C, Oranger A, Papale M, Sallustio F, Simone S, Laviola L, Giorgino F, et al. Urinary miRNA-27b-3p and miRNA1228-3p correlate with the progression of kidney fibrosis in diabetic nephropathy. Sci Rep. 2019;9(1):11357.

7. Warren AM, Knudsen ST, Cooper ME. Diabetic nephropathy: an insight into molecular mechanisms and emerging therapies. Expert Opin Ther Targets. 2019;23(7):579-91.

8. Kaul P, Savu A, Nerenberg KA, Donovan LE, Chik CL, Ryan EA, Johnson JA. Impact of gestational diabetes mellitus and high maternal weight on the development of diabetes, hypertension and cardiovascular disease: a population-level analysis. Diabet Med. 2015;32(2):164-73. 
9. Gnudi L, Coward RJM, Long DA. Diabetic Nephropathy: Perspective on Novel Molecular Mechanisms. Trends Endocrinol Metabol. 2016;27(11):82030. https://doi.org/10.1016/j.tem.2016.1007.1002.

10. Umanath K, Lewis JB. Update on diabetic nephropathy: Core curriculum 2018. Am J Kidney Dis. 2018;71(6):884-95.

11. Marketou NP, Chrousos GP, Kanaka-Gantenbein C. Diabetic Nephropathy in Type 1 Diabetes: A Review of Early Natural History, Pathogenesis and Diagnosis. Diabetes/Metabol Res Rev. 2016. https://doi.org/10.1002/dmrr. 2841.

12. Campion CG, Sanchez-Ferras O, Batchu SN. Potential role of serum and urinary biomarkers in diagnosis and prognosis of diabetic nephropathy. Can J Kidney Health Dis. 2017;4:2054358117705371.

13. Simpson K, Wonnacott A, Fraser DJ, Bowen T. MicroRNAs in diabetic nephropathy: from biomarkers to therapy. Curr Diabetes Rep. 2016;16(3):35.

14. Voelker J, Berg PH, Sheetz M, Duffin K, Shen T, Moser B, Greene T, Blumenthal SS, Rychlik I, Yagil Y. Anti-TGF- $\beta 1$ antibody therapy in patients with diabetic nephropathy. J Am Soc Nephrol. 2017;28(3):953-62.

15. Pezzolesi MG, Satake E, Mcdonnell KP, Major M, Smiles AM, Krolewski AS. Circulating TGF- $\beta 1$-regulated miRNAs and the risk of rapid progression to ESRD in type 1 diabetes. Diabetes. 2015;64:3285-93.

16. Badal SS, Danesh FR. Diabetic nephropathy: emerging biomarkers for risk assessment. Diabetes. 2015;64(9):3063-5.

17. Smyth GK. limma: Linear Models for Microarray Data. In: Gentleman R, Carey VJ, Huber W, Irizarry RA, Dudoit S, editors. Bioinformatics and Computational Biology Solutions Using R and Bioconductor. edn. New York, NY: Springer New York; 2005. p. 397-420.

18. Irizarry RA, Hobbs B, Collin F, Beazer-Barclay YD, Antonellis KJ, Scherf U, Speed TP. Exploration, normalization, and summaries of high density oligonucleotide array probe level data. Biostatistics. 2003;4(2):249-64.

19. Szklarczyk D, Franceschini A, Wyder S, Forslund K, Heller D, Huerta-Cepas J, Simonovic M, Roth A, Santos A, Tsafou KP. STRING v10: protein-protein interaction networks, integrated over the tree of life. Nucleic Acids Res. 2014;43(D1):D447-52.

20. Paraskevopoulou MD, Vlachos IS, Karagkouni D, Georgakilas G, Kanellos I, Vergoulis T, Zagganas K, Tsanakas P, Floros E, Dalamagas T. DIANA-LncBase V2: indexing microRNA targets on non-coding transcripts. Nucleic Acids Res. 2016;44(D1):D231-8.

21. RANKIN MM, GUO L, BAKAJ I, Ho G, Rady B, Zhao S, Stahle PL, Ghosh A, Shi $Y$, Patel S, et al. Therapeutic Potential of EP3 Receptor Antagonists for Treatment of Noninsulin Dependent Diabetes Mellitus across Multiple Preclinical Models. Diabetes. 2018;67(Supplement 1):250 LB.

22. Truchan NA, Sandhu HK, Fenske RJ, Buchanan R, Moeller J, Reuter A, Harrington J, Kimple ME. Differential Effects of Prostaglandin E2 Production and Signaling through the Prostaglandin EP3 Receptor on Human Beta-cell Compensation. bioRxiv. 2019. https://doi.org/10.1101/670000.

23. Kimple ME, Keller MP, Rabaglia MR, Pasker RL, Neuman JC, Truchan NA, Brar HK, Attie AD. Prostaglandin E2 receptor, EP3, is induced in diabetic islets and negatively regulates glucose-and hormone-stimulated insulin secretion. Diabetes. 2013;62(6):1904-12.

24. Schaid MD, Wisinski J, Laudre E, Kimple M. The Molecular Mechanisms of Prostaglandin E2 Receptor 3 and its associated G protein, Gz, in the Pancreatic $\beta$-cell. FASEB J. 2017;31(1_supplement):619.616.

25. Reuter A, Wisinski JA, Peter D, Schaid MD, Fenske RJ, Kimple ME. A role for EP3 and its associated $G$ protein, $G_{z}$ in negatively regulating beta-cell function and mass in the context of insulin resistance and obesity. bioRxiv. 2019. https://doi.org/10.1101/671289.

26. Cui $H$, Shan $H$, Miao MZ, Jiang Z, Meng Y, Chen R, Zhang L, Liu Y. Identification of the key genes and pathways involved in the tumorigenesis and prognosis of kidney renal clear cell carcinoma. Sci Rep. 2020;10(1):1-10.

27. Sapienza C, Lee J, Powell J, Erinle O, Yafai F, Reichert J, Siraj ES, Madaio M. DNA methylation profiling identifies epigenetic differences between diabetes patients with ESRD and diabetes patients without nephropathy Epigenetics. 2011;6(1):20-8.

28. Hassouneh R, Nasrallah R, Zimpelmann J, Gutsol A, Eckert D, Ghossein J, Burns KD, Hébert RL. PGE2 receptor EP3 inhibits water reabsorption and contributes to polyuria and kidney injury in a streptozotocin-induced mouse model of diabetes. Diabetologia. 2016;59(6):1318-28.

29. Bonner-Weir S, Aguayo-Mazzucato C, Weir GC. Dynamic development of the pancreas from birth to adulthood. Ups J Med Sci. 2016;121(2):155-8.

30. Arreguin-Cano JA, Ayerdi-Nájera B, Tacuba-Saavedra A, Navarro-Tito N, Dávalos-Martínez A, Emigdio-Vargas A, Barrera-Rodríguez E, Blanco-García N,
Gutiérrez-Venegas G, Ventura-Molina E, et al. MMP-2 salivary activity in type 2 diabetes mellitus patients. Diabetol Metabol Syndrome. 2019;11(1):113.

31. Stabouli S, Kotsis V, Maliachova O, Printza N, Chainoglou A, Christoforidis A, Taparkou A, Dotis J, Farmaki E, Zafeiriou D. Matrix metalloproteinase $-2,-9$ and arterial stiffness in children and adolescents: the role of chronic kidney disease, diabetes, and hypertension. Int J Cardiol Hypertension. 2020;4: 100025.

32. Preil SAR, Thorsen A-SF, Christiansen AL, Poulsen MK, Karsdal MA, Leeming DJ, Rasmussen LM. Is cardiovascular disease in patients with diabetes associated with serum levels of MMP-2, LOX, and the elastin degradation products ELM and ELM-2? Scand J Clin Lab Invest. 2017:77(7):493-7.

33. Tsukita S, Yamada T, Takahashi K, Munakata Y, Hosaka S, Takahashi H, Gao J, Shirai Y, Kodama S, Asai Y, et al. MicroRNAs 106b and 222 improve hyperglycemia in a mouse model of insulin-deficient diabetes via pancreatic B-cell proliferation. EBioMedicine. 2017:15:163-72.

34. Muendlein A, Geiger K, Leiherer A, Saely C, Ebner J, Brandtner E-M, Zanolin D, Vonbank A, Mader A, Larcher B. Correlation between circulating micrornas and chronic kidney disease in patients with and without type 2 diabetes. Atherosclerosis. 2018;275:e188.

35. Muendlein A, Geiger $K$, Leiherer A, Saely $\mathrm{CH}$, Fraunberger $\mathrm{P}$, Drexel $\mathrm{H}$. Evaluation of the associations between circulating microRNAs and kidney function in coronary angiography patients. Am J Physiol-Renal Physiol. 2020; 318(2):F315-21.

36. Hamilton MJ, Young $M$, Jang $K$, Sauer $S$, Neang VE, King AT, Girke T, Martinez E. HOTAIRM1 IncRNA is downregulated in clear cell renal cell carcinoma and inhibits the hypoxia pathway. Cancer Lett. 2020;472:50-8.

37. Lang IM. Treprostinil for severe inoperable chronic thromboembolic pulmonary hypertension. J Thrombosis Haemostasis. 2007;5(3):483-9.

38. Simonneau G. Treprostinil, a prostacyclin analogue, in pulmonary arterial hypertension associated with connective tissue disease. Chest. 2004;126(2): $420-7$.

39. Shindo H, Tawata M, Yokomori N, Hosaka Y, Onaya T. Iloprost decreases urinary albumin excretion rate in patients with diabetic nephropathy. Diabetes Res Clin Pract. 1993;21(2-3):115-22.

40. Leon P, Alexander PP, Joshua C, Iddo P, Veronica V, Antonella M. Captopri as an Adjuvant to Temozolomide Prolongs Survival in a Rat Intracranial Gliosarcoma Model via Downregulation of Matrix Metalloproteinase-2. Neurosurgery. 2019;66(810):169

\section{Publisher's Note}

Springer Nature remains neutral with regard to jurisdictional claims in published maps and institutional affiliations.

Ready to submit your research? Choose BMC and benefit from:

- fast, convenient online submission

- thorough peer review by experienced researchers in your field

- rapid publication on acceptance

- support for research data, including large and complex data types

- gold Open Access which fosters wider collaboration and increased citations

- maximum visibility for your research: over $100 \mathrm{M}$ website views per year

At $\mathrm{BMC}$, research is always in progress.

Learn more biomedcentral.com/submission 